\title{
Accuracy modulating mutations of the ribosomal protein S4-S5 interface do not necessarily destabilize the rps4-rps5 protein-protein interaction
}

\author{
HARITHA VALLABHANENI and PHILIP J. FARABAUGH \\ Program in Molecular and Cell Biology, Department of Biological Sciences, University of Maryland Baltimore County, Baltimore, Maryland \\ 21250, USA
}

\begin{abstract}
During the process of translation, an aminoacyl tRNA is selected in the A site of the decoding center of the small subunit based on the correct codon-anticodon base pairing. Though selection is usually accurate, mutations in the ribosomal RNA and proteins and the presence of some antibiotics like streptomycin alter translational accuracy. Recent crystallographic structures of the ribosome suggest that cognate tRNAs induce a "closed conformation" of the small subunit that stabilizes the codonanticodon interactions at the A site. During formation of the closed conformation, the protein interface between rpS4 and rpS5 is broken while new contacts form with rpS12. Mutations in rpS12 confer streptomycin resistance or dependence and show a hyperaccurate phenotype. Mutations reversing streptomycin dependence affect rpS4 and rpS5. The canonical rpS4 and rpS5 streptomycin independent mutations increase translational errors and were called ribosomal ambiguity mutations (ram). The mutations in these proteins are proposed to affect formation of the closed complex by breaking the rpS4-rpS5 interface, which reduces the cost of domain closure and thus increases translational errors. We used a yeast two-hybrid system to study the interactions between the small subunit ribosomal proteins rpS4 and rpS5 and to test the effect of ram mutations on the stability of the interface. We found no correlation between ram phenotype and disruption of the interface.
\end{abstract}

Keywords: Yeast two-hybrid; translational accuracy; ribosomal proteins; protein-protein interaction; RNA-protein interaction

\section{INTRODUCTION}

The accuracy of protein synthesis is critical to cellular growth and survival. Errant proteins can have deleterious effects on the cell so protein synthesis uses many mechanisms to minimize errors. Missense errors can result from the mischarging of tRNAs (Ibba and Soll 1999) or can happen during translation when the ribosome allows a codon to be recognized by an incorrect tRNA (Kurland et al. 1996). Other translational errors occur when a tRNA decodes out of the normal reading frame (a frameshift error), or protein synthesis aborts by the dissociation of the peptidyl-tRNA from the ribosome (Kurland et al. 1996). Translation errors occur infrequently, with the frequency of missense errors, for example, ranging between $10^{-3}$ and

Reprint requests to: Phillip J. Farabaugh, Program in Molecular and Cell Biology, Department of Biological Sciences, University of Maryland Baltimore County, Baltimore, MD 21250, USA; e-mail: farabaug@umbc. edu; fax: (410) 455-3875.

Article published online ahead of print. Article and publication date are at http://www.rnajournal.org/cgi/doi/10.1261/rna.1530509.
$10^{-4}$ per codon (Kramer and Farabaugh 2007, and references therein).

Intrinsic tRNA stability is insufficient to explain the accuracy of protein synthesis (Uhlenbeck et al. 1971; Thompson and Stone 1977; Grosjean et al. 1978). The energy difference between binding of correct (cognate) and near-cognate tRNAs, those having a single mismatch with the codon, of only $3 \mathrm{kcal} / \mathrm{mol}$ would predict $10^{-2}$ errors per codon, a much higher error frequency than is observed (Ogle and Ramakrishnan 2005). The kinetic proofreading model (Hopfield 1974; Ninio 1975) suggested that repeated discrimination in a two-step process separated by an irreversible intermediate could square the 100-fold selectivity based on tRNA stability differences. Under this model, repeated discrimination before and after GTP hydrolysis on EF-Tu would provide 10,000-fold selectivity, in the range observed in vivo (Thompson 1988). Recent kinetic analysis has suggested that discrimination on the basis of differential tRNA stability cannot explain the observed discrimination (for review, see Rodnina et al. 2005). Instead, the most critical parameter appears to be 
the rate of activation of the intrinsic GTPase of EF-Tu, which is almost three orders of magnitude faster for correct complexes. The ribosome cannot maximize discrimination using differences in tRNA stability because it proceeds to the next step in the reaction sequence too rapidly to allow the full thermodynamic difference to be achieved (Gromadski et al. 2006).

Potapov (1982) proposed that the ribosome recognizes the stereochemical correctness of the geometry of codonanticodon base pairing in the ribosomal aminoacyl-tRNA (A) site. Recent crystallographic studies support this model. The A site of the ribosome appears to recognize the shape of cognate tRNA•mRNA complexes (Carter et al. 2000; Ogle et al. 2001). The A site consists of parts of helix 18 (h18) from the body of the small subunit, h44 and h34 from the head and ribosomal protein rpS12 (Moazed and Noller 1990; O'Connor et al. 1995). The $16 \mathrm{~S}$ rRNA bases A1492 and A1493 of h44 and G530 of h18 form the floor of the A site (Wimberly et al. 2000). When a cognate tRNA binds in the A site, A1492 and A1493 flip out from positions stacked within h44 and G530 changes from a syn to an anti conformation. These changes allow the nucleotides to interact with each other and/or the minor groove of the codon-anticodon helix by hydrogen boding to ribose 2'-OH groups (Carter et al. 2000; Ogle et al. 2001). A1493 bridges between the codon and anticodon 2'-OH groups of the first base pair and G530 and A1492 form an N3-N3 hydrogen-bonded pair that bridges the second codon-anticodon base pair. These interactions require the unique geometry of a Watson-Crick base pair (Leontis et al. 2002). G530 also bonds to the $2^{\prime}-\mathrm{OH}$ of the third or wobble nucleotide of the anticodon while C1054 stacks on the base of the wobble codon nucleotide (Ogle et al. 2001). These interactions do not require Watson-Crick geometry and so do not discriminate against some non-WatsonCrick pairs (e.g., G-U).

Local changes in the decoding site induce global changes of the small subunit, resulting in change from an "open" to a "closed" conformation in which the elements of the shoulder domain are brought in close proximity to the codon-anticodon helix in the decoding center (Ogle et al. 2002). The closed conformation of the ribosome involves interactions between the $30 \mathrm{~S}$ subunit and both the tRNA and EF-Tu that stabilize the ternary complex and cause EF-Tu to interact with GTPase activation center (GAC) of the large subunit. The GAC interaction explains the great acceleration of GTPase activation for cognate but not nearcognate EF-Tu ternary complexes and therefore the preference of the ribosome for cognate tRNAs (Rodnina et al. 2005). During the closure of the subunit, new interactions form between the ribosomal protein rpS12 and 16S rRNA, especially with $\mathrm{h} 44$ and $\mathrm{h} 27$, while polar interactions at the interface between proteins $\mathrm{rpS} 4$ and $\mathrm{rpS} 5$ are disrupted (Ogle et al. 2002). How do interactions deep in the decoding center cause the large-scale change in ribosome structure?
What roles, if any, are played by changes in $\mathrm{rpS4}$, rpS5, and rpS12 protein-protein and protein-RNA interactions?

Clues to the answers to these questions come from studies showing how aminoglycoside antibiotics, such as streptomycin, induce misreading of the genetic code (Davies et al. 1964; Gorini and Kataja 1964). Streptomycin resistant mutations mapping to rpS12 show three different phenotypes: streptomycin resistant (SmR) (Ozaki et al. 1969), streptomycin dependent (SmD) (Birge and Kurland 1969) or streptomycin pseudodependent (SmP) (Zengel et al. 1977; Ruusala et al. 1984). All three grow in the presence of usually lethal concentrations of the antibiotic, but $\mathrm{SmD}$ mutants require the antibiotic for survival and the growth of SmP mutants improves in its presence. These mutants are all hyperaccurate with $\mathrm{SmD}$ showing the fewest translational errors and SmP being intermediate. Streptomycin-independent revertants of SmD mutants were mapped to rpS4 and rpS5 (Birge and Kurland 1970; Deusser et al. 1970; Kreider and Brownstein 1971; Stoffler et al. 1971; Donner and Kurland 1972). Rosset and Gorini (1969) showed that compensatory rpS4 and rpS5 mutations themselves conferred an error prone phenotype and called them ribosomal ambiguity ( $\mathrm{ram}$ ) mutants. These mutations were explained as reducing the accuracy to a level at which addition of streptomycin was no longer required for viability.

Most of the SmD-compensatory mutations are at or near to the interface of rpS4 and rpS5 proteins (Maisnier-Patin et al. 2002; Ogle et al. 2002). Given that the transition from the open to the closed conformation induced by cognate tRNA in the A site disrupts the interface of rpS4 and rpS5 (Ogle et al. 2002), the idea that these mutations alter the interface region suggested that they might destabilize the interaction and facilitate domain closure even in the absence of cognate tRNA (Ogle et al. 2002). Because the cost of domain closure would be reduced, near cognate tRNAs would tend to be accepted into the A site more readily, leading to the ambiguity phenotype. It was shown recently, however, that one rpS4 ram mutation increased misreading at only a subset of near cognate codons rather than with all of them (Kramer and Farabaugh 2007), which contradicts the domain closure model. In Salmonella enterica Serovar Typhimurium (S. typhimurium), moreover, it was shown that some of SmD-compensatory rpsD mutations are themselves hyperaccurate, suggesting that the compensatory mutations to hyperaccuracy need not be ram (Bjorkman et al. 1999). It is unclear how the growth restrictive phenotype of one restrictive mutation could be compensated for by a second restrictive mutation. The recent description of an rpS5 (G28D) ram mutation affecting a residue remote from the rpS4/rpS5 interface (Kirthi et al. 2006) suggested there are ways to induce a ram phenotype other than by disrupting the rpS4-rpS5 interface.

In this study we directly tested the correlation between the SmD-compensatory phenotype and the stability of the 
rpS4-rpS5 interface using the yeast two-hybrid system. We tested classical ram mutations in rpS4 and rpS5 and several of the compensatory mutations isolated in S. typhimurium (Bjorkman et al. 1999). Our results show that only some of the mutations tested destabilize the rpS4-rpS5 interaction. Destabilization does not correlate with the error phenotype of the mutations, that is, destabilizing mutants can be either error prone or hyperaccurate, as can be the nondestabilizing mutations. This lack of correlation suggests that the mutations may affect translational accuracy by a mechanism other than destabilizing this protein-protein interface. Alternative explanations of the effect of the rpS4 and rpS5 mutations will be discussed.

\section{RESULTS}

\section{Yeast two-hybrid analysis confirms rpS4-rpS5 interaction}

The two ribosomal proteins, $\mathrm{rpS} 4$ and $\mathrm{rpS} 5$, reside in adjacent positions on the solvent face of the $30 \mathrm{~S}$ subunit (Fig. 1). Each protein makes extensive contacts with the $16 \mathrm{~S}$ rRNA involving over $35 \%$ of the solvent-accessible surfaces and areas of over 2871 and $2010 \AA^{2}$ for rpS4 and rpS5, respectively. The proteins interact with each other over a

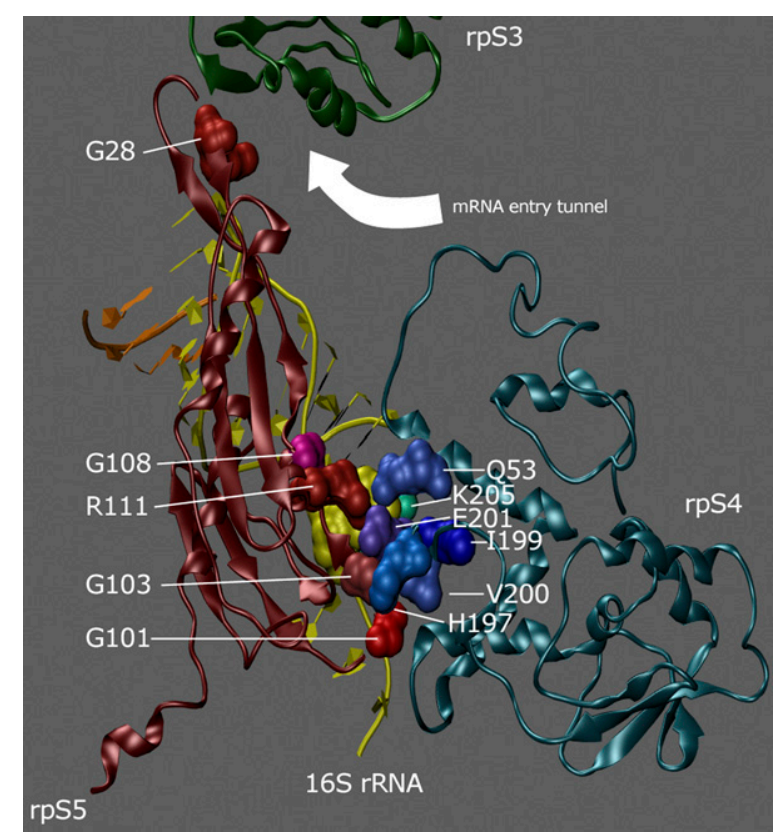

FIGURE 1. Interactions at the rpS4•rpS5 protein-protein interface. Location of mutant residues analyzed in this work. The E. coli rpS4 (blue) and rpS5 (red) proteins and residues 5-25 of 16S rRNA are shown in cartoon form (PDB 2AVY, visualized using Visual Molecular Dynamics and MegaPOV); along with rpS3 (green cartoon), they form the ribosome helicase center through which the mRNA enters the ribosome (arrow). The mutant residues (labeled in white) are shown in space-filling format in shades of green for $\mathrm{rpS} 4$ and red for rpS5. significantly smaller region corresponding to $362 \AA^{2}$ of rpS4 and $346 \AA^{2}$ rpS5, which correspond to $3.0 \%$ and $4.1 \%$ of their solvent-accessible surface.

To study the interactions between the two small subunit ribosomal proteins, rpS4 and $\operatorname{rpS5}$, we used the Matchmaker LexA yeast two-hybrid system (Clontech). In the Matchmaker system the pGilda plasmid encodes the DNA binding domain (BD) of the LexA bacterial repressor protein (LexA) and the pB42AD plasmid encodes an 88residue acidic peptide (B42) (Ma and Ptashne 1987) activation domain (AD) tagged with the Haemophilus influenzae hemagglutinin (HA) epitope. The test proteins are fused in frame to the DNA-BD and AD, and if they interact they form a transcriptional activator that results in activation of the reporter genes. The LexA system uses two reporter genes (LEU2 and lacZ) under the control of multiple LexA operators to eliminate false positives; the assay for LEU2 activity tests for growth of transformed yeast cells in the absence of leucine (-Leu plates) and the assay for $\operatorname{lac} Z$ measures the specific activity of its encoded product, $\beta$-galactosidase.

We cloned the entire rpS4 coding region of $r p s D$ into $\mathrm{pB} 42 \mathrm{AD}$ (pB42AD-rpsD) and the rpS5 coding region of $r p s E$ into pGilda (pGilda-rpsE) to test for potential interactions. A yeast strain cotransformed with vectors pGilda and $\mathrm{pB} 42 \mathrm{AD}$ lacking an insert was used as a negative control. As a further control, we tested for activation of the reporter genes that did not require both inserts by transforming the yeast reporter strain with pGilda-rpsE or pB42AD-rpsD singly or in combination with the corresponding empty vector.

Cells transformed with the pB42AD-rpsD and pGildarpsE expressed significant amounts of $\beta$-galactosidase (averaging $38 \pm 6.3$ units) and about fivefold above the background for all negative control transformants (Fig. 2A; data not shown). The cotransformants with pB42AD-rpsD and pGilda-rpsE also grew on -Leu plates (Fig. 3, top row) but the negative controls did not (Fig. 3, bottom three rows). These data show that whereas neither the $r p s D$ nor $r p s E$ protein fusions could by themselves activate the lac $Z$ and LEU2 reporter genes, the combination of the two significantly stimulated both genes. Though qualitative, the LEU2 reporter appears to be much more sensitive than the lac $Z$ reporter. These data show that the yeast two-hybrid assay confirms the interaction between the two proteins.

\section{Several ram and SmD-compensatory mutants of rpS5 fail to destabilize the rpS4-rpS5 interaction in vivo}

In the ribosome the two proteins are constrained to interact at juxtaposed interface regions. Even a relatively weak interaction between the two proteins could have a significant effect on ribosome function since this juxtaposition would result in an artificially high apparent binding constant since the rate of association of the two proteins 


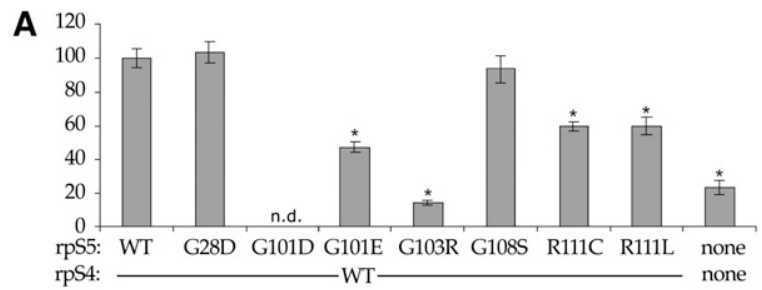

B $\mathrm{rpS5}^{+}$G28D G101E G103R G108S R111C R111L C *

FIGURE 2. Analysis of interactions between rpS4 and rpS5 mutants using $\beta$-galactosidase activity. (A) $\beta$-Galactosidase assays. Each column represents the percent $\beta$-galactosidase activity relative to the wild type. The results are average of at least three independent assays done with three to four transformants each time. The error bars represent standard error of the mean. (B) Western blot to determine the relative levels of protein expressed. Extracts from yeast transformants were made by vortexing with glass beads in lysis buffer as described in Materials and Methods. Twenty micrograms of total protein for each sample were loaded and detected using LexA antibody. Detection with a $\beta$-tubulin (asterisk) antibody controlled for loading.

would be essentially instantaneous. It was by no means certain that the yeast two-hybrid assay would be sufficiently sensitive to allow detection of such a weak interaction but the results clearly indicate that the two proteins do associate in vivo. It is not necessarily true that the two proteins would interact in this heterologous system at the same surface residues used in the ribosome. We therefore extended the analysis to test the effect of known SmDcompensatory mutant forms of rpS4 and rpS5 to determine if they altered the efficiency of the interaction, as they were predicted to do by Ogle et al. (2002).

We constructed $r p s E$ mutants altering five residues located in or very near the rpS4-rpS5 interface (Gly101, Gly103, Gly108, and Arg111) or located outside the interface (G28). The mutations rpsE-G103R and rpsE-R111L affecting rpS5 were identified in Escherichia coli as suppressors of a SmD mutant form of $r p s L$ and as classical ram mutations (Ito and Wittmann 1973). In Bacillus subtilis rpsE-G103R, rpsE-R111C, and $r p s E-R 111 \mathrm{H}$ were isolated using an identical suppressor selection (Inaoka et al. 2001). In the absence of the rpsL mutation, $r p s E$ $R 111 H$ conferred a ram phenotype while rpsE-R111C and $r p s E-G 103 R$ were lethal so their accuracy phenotype was not determined. Similarly, rpsE-G101D and rpsE-R111C were isolated as spontaneous streptomycin independent mutants from rpsL SmD mutants in S. typhimurium; however, their accuracy phenotype was not determined (Bjorkman et al. 1999). We also tested the effect of an rpsEG101E mutation predicted to be similar in phenotype to $r p s E-G 101 D$. The mutation $r p s E-G 108 S$ resulted in fast growth in presence of the restrictive rpsL mutation in $S$. typhimurium (Bjorkman et al. 1999). Another mutation
G28D (Kirthi et al. 2006) is shown to have a ram-like phenotype and maps away from the predicted rpS4 and rpS5 interface.

Expression of rpS5 and the mutants as fusions to LexA protein in pGilda vector was first verified by Western blot analysis using anti-LexA antibody. All the hybrid proteins are expressed well except for rpsE-G103R (Fig. 2B), which shows a lower intensity band compared with the $\beta$-tubulin control. A lower concentration of the expressed protein in vivo might reduce activation in the yeast two-hybrid system.

All the rpS5 constructs were cotransformed with a $\mathrm{pB} 42 \mathrm{AD}$ vector expressing the wild-type rpS4 protein and tested for activation of reporter genes lacZ and LEU2. The $\beta$-galactosidase activity of the double transformants showed that one of the five mutations affecting the interface region ( $r$ sE-G108S) had activity that was not significantly different from the wild type (Fig. 2A). The rpsE-G28D mutant, affecting a residue outside the interface, also had activity indistinguishable from wild type. Four mutations, rpsE-G101E, rpsE-G103R, rpsE-R111C, and rpsE-R111L, expressed significantly reduced activity; the expression of the rpsE-G103R mutant was statistically indistinguishable from the negative controls. The lower activation dependent on $r p s E-G 103 R$ could result from its poor expression in vivo, or it could reflect its disruption of the interface.

As a further test of the affect of the rpS5 mutations on the protein-protein interaction, we determined their effect on activation of the LEU2 reporter using the serial dilution method. The LEU2 assay was qualitatively similar to the $\beta$-galactosidase results. In agreement with the $\beta$-galactosidase assay, growth on -Leu plates for both rpsE-G28D and rpsEG108S was indistinguishable from wild type. The LEU2 phenotypes of putative destabilizing mutants are more

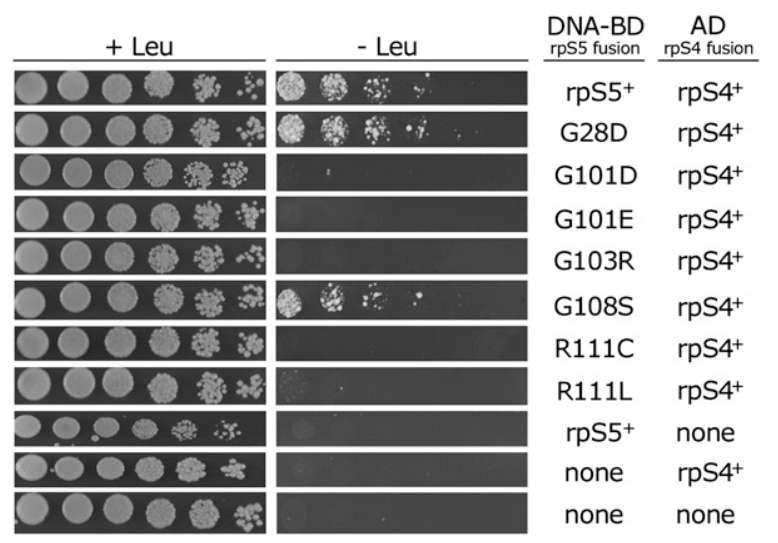

FIGURE 3. Analysis of interactions between $\mathrm{rpS} 4$ and rpS5 mutants by Leucine protrophy. The fusion proteins encoded in the DNA-BD and $\mathrm{AD}$ vectors are shown on the right. Ten microlitres of fivefold serial dilutions of cells are spotted on $\mathrm{SD} / \mathrm{gal} / \mathrm{raf}$ plates with Leu $(+$ Leu $)$ and without Leu ( - Leu) and incubated at $30^{\circ} \mathrm{C}$ for 5 days. 
extreme than their lacZ phenotypes. The mutants G101E, R111C, and R111L showed significantly decreased but nonzero $\beta$-galactosidase activity that was nonetheless significantly higher than the negative control. These mutants and two others (G101D and G103R) showed no growth in the LEU2 assay, similar to the negative controls (Fig. 3). The LEU2 reporter again appears to report much more sensitively a loss of interaction. We presume that the activity of the enzyme encoded by $L E U 2$ provided by these mutants falls below a critical level sufficient to support growth; that level may be significantly higher than the activity of a null strain despite providing the same growth phenotype. The two reporters agree in showing that the G28D and G108S mutants do not appear to destabilize the rpS4-rpS5 interaction.

In order to test if the yeast two-hybrid results are due to interactions between the two proteins and not due to activation of the reporter genes that bypasses the need for a protein-protein interaction we confirmed these results using a coimmunoprecipitation assay. We used a HA-tag Co-IP kit (Pierce), in which an antihemagglutinin (HA) antibody is conjugated to agarose beads, to pull down the HA-tagged B42AD-rpS4 chimeric protein. LexA-rpS5 chimeric protein coimmunoprecipitating with $\mathrm{rpS} 4$ was detected using an anti-LexA antibody. As negative controls we tested coimmunoprecipitation with anti-HA antibody in extracts of yeast transformed with pB42AD-rpS4 and pGilda (Fig. 4) or pB42-AD and pGilda-rpS5 (data not shown); neither control showed substantial coimmunoprecipitation of the LexA protein expressed from pGilda showing that any coimmunoprecipitation in other samples was caused by an interaction between $\mathrm{rpS} 4$ and rpS5. The amount of LexA-rpS5-G28D and LexA-rpS5-G108S pulled down by B42AD-rpS4 was indistinguishable from the wildtype LexA-rpS5 (Fig. 4). For all other mutant proteins, the amount of protein pulled down was from two- to fivefold less than wild type (Fig. 4). These data are consistent with the results of the lac $Z$ and $L E U 2$ yeast two-hybrid assays and confirm that the rpsE-G28D and rpsE-G108S mutations do not disrupt the rpS4-rpS5 protein-protein interaction.

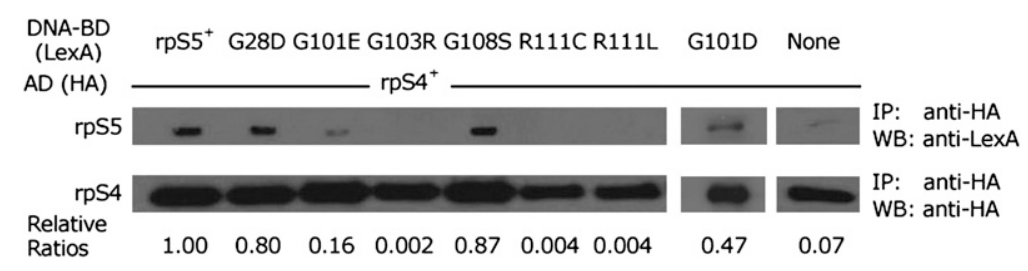

FIGURE 4. Analysis of interactions between $\mathrm{rpS} 4$ and $\mathrm{rpS} 5$ and mutants by coimmunoprecipitation assays. Extracts containing $40 \mu \mathrm{g}$ of protein were incubated with anti HA agarose slurry $(6 \mu \mathrm{L})$ overnight at $4^{\circ} \mathrm{C}$ washed and eluted in $25 \mu \mathrm{L}$ of nonreducing sample buffer. Equal amounts $(10 \mu \mathrm{L})$ of washed and eluted samples were loaded in duplicate and detected by anti-LexA (above) or anti-HA antibody (below). Transformants with pB42AD-S4 cotransformed with pGilda vector is shown as a negative control. G101D was expressed at equivalent levels to the wild-type protein (data not shown).

\section{The accuracy phenotypes of rpS4 mutants do not correlate with their effect on the rpS4-rpS5 interaction}

In S. typhimurium, $r p s D$ mutations that compensate for hyperaccurate $\mathrm{SmD}$ rpsL mutations need not necessarily be error prone. The $r p s D$ mutations Q53L, Q53P, I199N, $\Delta$ V200, E201* (a nonsense mutation at position 201), $\mathrm{K} 205 \mathrm{~N}$, and $\mathrm{K} 205 \mathrm{~T}$ were isolated in S. typhimurium (Bjorkman et al. 1999). Among these mutants, Q53P, I199N, K205N, and K205T are all error prone, whereas Q53L, $\Delta$ V200, and E201*, despite suppressing the Strdependence of hyperaccurate rpsL mutations, are themselves also hyperaccurate. Consistent with this phenotype, the hyperaccurate $r p s D$ mutations in an $r p s L^{+}$background also confer weak streptomycin resistance rather than the sensitivity shown by the rest of the mutations (Bjorkman et al. 1999). We were also interested in looking at the effect of H197, which showed interactions with rpS5-G101 and rpS5-G103 in the crystal structure and has been inferred to be error prone (Ogle et al. 2002). We substituted H197 with Ala to study its effect on the interface.

The error-prone mutations $r p s D-12, r p s D-14$, and $r p s D$ 16 cause C-terminal truncations of rpS4 (Olsson and Isaksson 1979). In rpsD-12, 5 nucleotides (nt) (528-532) are deleted and in $r p s D-14,1 \mathrm{nt}$ is inserted after nucleotide 535, resulting in the 205 amino acid wild-type protein being truncated to 180 and 182 amino acids, respectively. In each case, the mutant proteins have short C-terminal extensions not present in the wild type. The protein is truncated to 169 amino acids in $r p s D-16$ because of the tryptophan at position 170 is changed to a stop codon (Dahlgren and Ryden-Aulin 2000; Ogle et al. 2002).

Plasmids encoding the wild type and all the mutant forms of pB42AD-rpS4 were transformed along with the pGildarpsE vector, expressing the LexA-rpS5 protein. The mutants fell into three classes with respect to $\beta$-galactosidase activity (Fig. 5A). The truncation mutations, $r p s D-12, r p s D-14$, and $r p s D-16$, had activity statistically indistinguishable from the negative controls, indicating that they completely eliminate the rpS4-rpS5 interaction. Three other mutations stimulated slightly higher activity, but significantly less than wild type ( $r p s D-Q 53 P, r p s D-I 199 N$, and $r p s D-\Delta V 200)$, suggesting that they reduce the stability of the interaction. The other five mutant proteins stimulated activity statistically indistinguishable from wild type (rpsD-Q53L, rpsD-H197A, $r p s D-E 201^{*}, r p s D-K 205 N$, and $r p s D-$ K205T), indicating that they do not destabilize the interaction. Western blot analysis showed equivalent expression of each mutant protein with the exception of $r p s D-14$, which showed a less intense 
A

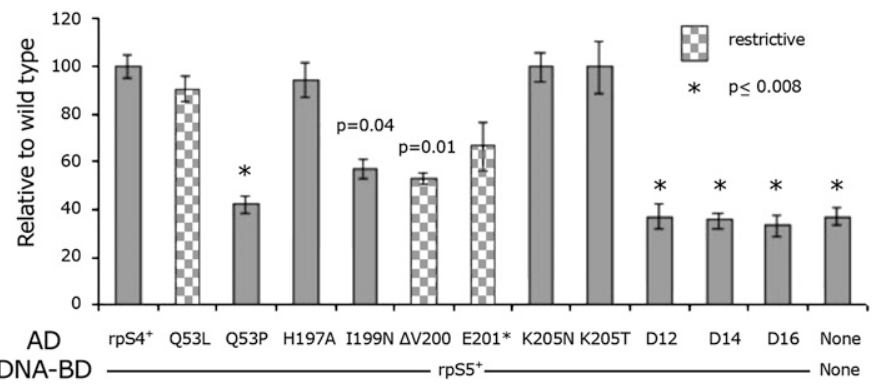

B
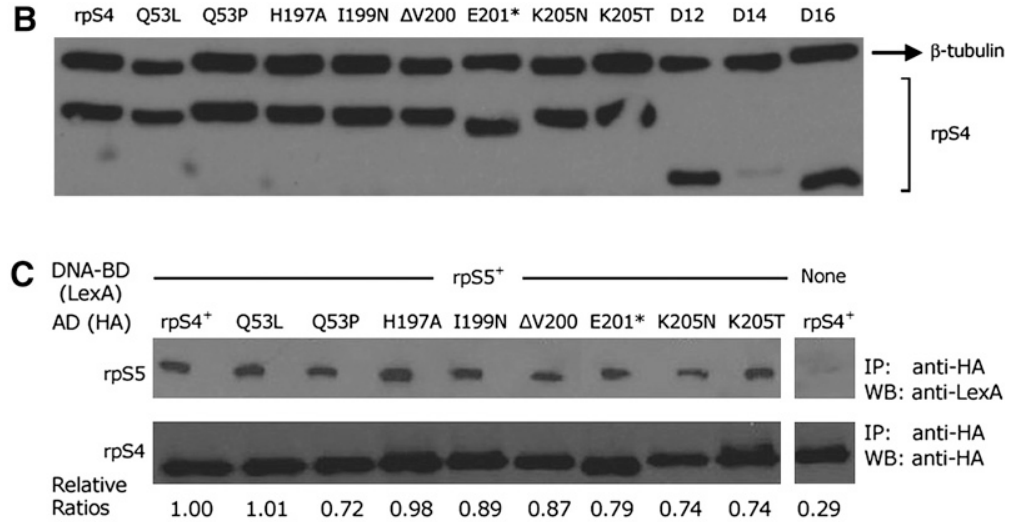

\section{D}
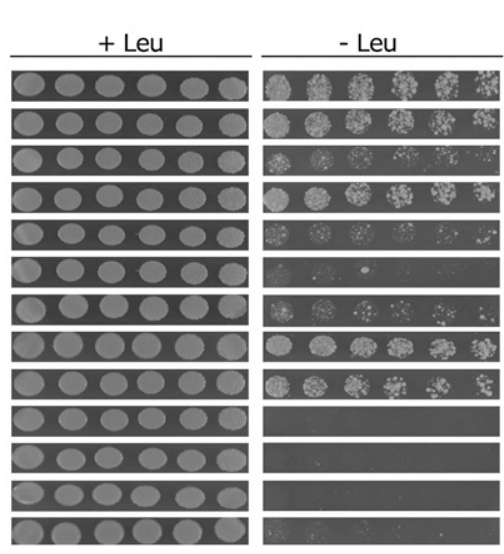

\begin{tabular}{cc}
$\begin{array}{cc}\text { DNA-BD } \\
\text { rpS5 fusion }\end{array}$ & $\begin{array}{c}A D \\
\text { rpS4 fusion }\end{array}$ \\
\cline { 1 - 1 } rpS5 $^{+}$ & rpS4 \\
rpS5 $^{+}$ & Q53L \\
rpS5 $^{+}$ & Q53P \\
rpS5 $^{+}$ & H197A \\
rpS5 $^{+}$ & I199N \\
rpS5 $^{+}$ & $\Delta V 200$ \\
rpS5 $^{+}$ & E201* \\
rpS5 $^{+}$ & K205N \\
rpS5 $^{+}$ & K205T \\
rpS5 $^{+}$ & D12 \\
rpS5 $^{+}$ & D14 \\
rpS5 $^{+}$ & D16 \\
none & none
\end{tabular}

FIGURE 5. Analysis of interaction between rpS5 and rpS4 mutants. (A) Western analysis to determine relative the expression of $\mathrm{rpS} 5$ and $\mathrm{rpS} 4$ mutants. Twenty micrograms of total protein for each sample were loaded and detected using Anti-HA antibody. $\beta$-Tubulin antibody was used as a loading control. $(B)$ Analysis of interactions between rpS5 and the mutants of rpS4 mutants by $\beta$-galactosidase assays performed as in Figure 2B. (C) Coimmunoprecipitation of wild-type $\mathrm{rpS} 4$ by wild-type and mutant forms of rpS5. Extracts containing $50 \mu \mathrm{g}$ of protein were immunoprecipitated and analyzed as in Figure 4. (D) Analysis of interactions between $\mathrm{rpS} 5$ and $\mathrm{rpS} 4$ by Leucine prototrophy was performed as in Figure 3.

band (Fig. 5B). All truncated proteins, $r p s D-E 201^{*}, r p s D-12$, $r p s D-14$, and $r p s D-16$, show proteins of smaller mass than the wild type. These data show that differences in $\beta$-galactosidase activity do not result from lack of expression, with the exception of $r p s D-16$. Overall, the lac $Z$ expression data fail to correlate with the accuracy phenotype. The three hyperaccurate mutants, $r p s D-Q 53 L, r p s D-\Delta V 200$, and $r p s D$ $E 201^{*}$ fall into two different classes (wild type and reduced activity), and the remaining error-prone mutants fall into all three classes.

Coimmunoprecipitation assays were generally consistent with the $\beta$-galactosidase activity. As with the rpS5 mutants described above, complex formation between rpS5 and mutant forms of rpS4 was analyzed by immunoprecipitating the HA-tagged rpS4 fusion proteins, separating the immunoprecipitated proteins on SDS-PAGE and probing duplicate samples with antiHA or anti-LexA antibody. Coimmunoprecipitation of two rpS4 mutant proteins, Q53L and H197A, was indistinguishable from wild-type protein (Fig. 5C); these two mutants promoted wild-type $\beta$-galactosidase activity. The other mutants analyzed all showed coimmunoprecipitation somewhat less than for wild type (Fig. 5C). The extent of coimmunoprecipitation of mutant forms of rpS4 was much higher in general than for rpS5 mutants. We suspect that the difference may derive from the fact that the rpS4 fusion proteins are expressed from a multicopy plasmid while the rpS5 fusion proteins are expressed from a single-copy plasmid. An excess of mutant forms of rpS4 may drive the capture wild-type rpS5 present in low concentration by mass action despite a low affinity interaction.

The effect of the rpS4 mutants on the LEU2 phenotype generally agreed with the $\beta$-galactosidase assays (Fig. 5D). The truncation mutants $r p s D-12, r p s D$ 14 , and $r p s D-16$ showed no growth, similar to that of the negative control. The intermediate class mutants, $r p s D$ Q53P, $r p s D-I 199 N$, and $r p s D-\Delta V 200$, all showed evidence of slightly more growth than the negative control or the truncations. The pseudo-wild-type class mutations, $r p s D-Q 53 L, \quad r p s D-$ H197A, rpsD-K205N, and rpsD-K205T, showed growth indistinguishable from the wild type. The lone exception was the mutation $r p s D$ E201*, a member of the pseudowild-type class, which showed reduced growth similar to the intermediate class mutations; this result is more consistent with the results of coimmunoprecipitation, and suggests that the $\beta$-galactosidase assay may have overestimated the activation by this mutant protein. Although the overall results of the three analyses of rpS4 mutants is less consistent than the analysis of the 
rpS5 mutants, the correlation especially of the lac $Z$ and LEU2 reporter assays is very strong and inconsistent with the predictions of the domain closure model.

\section{DISCUSSION}

When an aminoacyl-tRNA•EF-Tu•GTP ternary complex in the A site is not cognate for the mRNA codon (a near or noncognate) the ribosome is in an "open" conformation such that the aminoacyl-tRNA can readily dissociate. A cognate ternary complex occupying the A site induces a shift to the "closed" complex, reducing its dissociation from the ribosome and activating the GTPase of EF-Tu by juxtaposing it to the GTPase activating center of the $60 \mathrm{~S}$ ribosomal subunit. The shift from the open to the closed complex involves a large-scale rearrangement of the ribosome, including the disruption of the rpS4-rpS5 proteinprotein interface and formation of contacts between $\mathrm{rpS} 12$ and 16S rRNA (Ogle et al. 2002). Streptomycin-dependent $(\mathrm{SmD})$ mutants of $\mathrm{rpS12}$, encoded by $r p s L$, appear to interfere with closed complex formation by disrupting the required $\mathrm{rpS} 12 \bullet 16 \mathrm{~S}$ rRNA contacts (Ogle et al. 2002). The resistance to adopting the closed conformation explains the hyperaccuracy of these restrictive mutants (res). Mutations affecting rpS4 and rpS5, encoded by $r p s D$ and $r p s E$, that compensate for this hyperaccuracy affect residues in or near their protein-protein interface. The fact that they alter the interface implied that they may compensate for the $r p s L$ mutations by destabilizing the rpS4-rpS5 interface, which would tend to facilitate the shift to the closed conformation; these mutations were expected to be error prone (ram).

Our yeast two-hybrid results show that some SmDcompensatory $r p s D$ and $r p s E$ mutations do disrupt the protein-protein interaction, but that others do not. We obtained a spectrum of results with some mutations having phenotypes equivalent to the wild type, some to the negative control and others that fall between the wild type and negative control. The pseudowild-type mutations include G28D and G108S in rpS5 and Q53L, H197A, E201*, $\mathrm{K} 205 \mathrm{~N}$, and K205T in rpS4. Among these, G108S in rpS5 and Q53L, E201*, K205N, and K205T in rpS4 are all known to compensate for a hyperaccurate $r p s L$ mutation. Under the domain closure model for suppression of the rpsL mutation, each of these mutations was expected to destabilize the rpS4•rpS5 interface. The fact that none of these mutations interfere with the protein-protein interaction is inconsistent with the model. However, their phenotype would also be consistent with the rpS4 and rpS5 interacting at different interfaces in this assay than they do in the ribosome. The evidence that the interaction involves residues at the interface used in the ribosome is that many other mutations do interfere with interaction. These include rpS4 mutations Q53P, I199N, E201*, D12, D14, and D16 and rpS5 mutations G101D, G101E, G103R,
R111C, and R111L. The fact that half of the point mutations tested interfere with the yeast two-hybrid interaction implies that the interaction involves the predicted interface.

Where tested, all of the rpS5 mutations tested confer a error-prone phenotype. Among the rpS4 mutations, there are eight known ram and three known res mutations. We find no correlation between the gross phenotype of the rpS4 mutations and the results of the yeast two-hybrid test; that is, there are ram and res mutations in both the pseudowild-type and reduced interaction classes. We, therefore, find no correlation between the breakage of interface between the proteins $\mathrm{rpS} 4$ and $\mathrm{rpS5}$ and the compensatory effects of the ram and res mutations in either protein.

The mutation rpsE-G28D in rpS5 is unlike the other available mutants in that rather than being isolated as a suppressor of an SmD rpsL mutation, it was isolated as a spectinomycin resistant mutation, though it also shows a ram phenotype (Kirthi et al. 2006). Its epistatic effect on hyperaccurate $r p s L$ mutations is unknown. The mutation alters a highly conserved residue in the loop 2 region where other spectinomycin resistant mutations are found (Kirthi et al. 2006). The site is outside the rpS4-rpS5 interface and the phenotype was proposed to be due to its indirect effect on the interface or due to conformational changes in loop 2 (Kirthi et al. 2006). Our data argue that the mutation does not disrupt the protein-protein interaction.

The fact that most compensatory mutations map to the interface region (Maisnier-Patin et al. 2002) suggests that the interface between these proteins might play a crucial role in maintaining translational accuracy. In addition, most of these SmD-compensatory mutations affect opposing residues in the rpS4-rpS5 interface, suggesting that they play an important role in accuracy. However, our yeast two-hybrid data suggest that their role might not explicitly involve their participation in the protein-protein interface. An alternative explanation might involve the interaction between each protein and the rRNA. For example, the ram mutants of rpS4 block its binding to rRNA in vitro suggesting that they alter a region critical to ribosome association (Daya-Grosjean et al. 1972; Allen and Noller 1989). The proteins rpS4, rpS5, and rpS12 contact $16 \mathrm{~S}$ rRNA residues near the decoding center, which comprises parts of three regions of the small subunit rRNA surrounding helix 18, helix 34, and helix 44 (O'Connor et al. 1997; Brodersen et al. 2002). Furthermore, parts of helix 18 region and the central pseudoknot of $16 \mathrm{~S}$ rRNA, consisting of helix 1 and 2, interact with rpS4 and rpS5 adjacent to or in the interface between the two proteins (Brodersen et al. 2002). Among the SmD compensatory mutants is an rRNA mutation in this interface region of the rRNA that also confers a ram phenotype (Allen and Noller 1991). The compensatory mutations in rpS4 and rpS5 might confer their accuracy phenotypes by altering the RNA interface. 
Thus, mutations in these proteins might affect the conformation of the surrounding RNA near the decoding region leading to either ram or res phenotypes. However, either ram or res mutants of rpS4 and rpS5 might induce the same conformational changes that could complement those changes in RNA due to the restrictive mutations in rpS12.

\section{MATERIALS AND METHODS}

\section{Yeast two hybrid}

The LexA Matchmaker yeast two-hybrid system (Clontech) was used for this study. The plasmids $\mathrm{pB} 42 \mathrm{AD}$, a high-copy $2 \mu$ plasmid carrying an activation domain and a HA tag, and pGilda, a unit copy CEN-ARS plasmid providing a LexA DNA binding domain, are used to make the fusion constructs. The protein expression in both the plasmids is under the control of inducible GAL1 promoter. The reporter strain is a Saccharomyces cerevisiae strain EGY48 (his3, trp1, ura3, LexAop-LEU2, p8op-lacZ) with a $L E U 2$ reporter gene integrated into the genome and a lacZ reporter gene on a plasmid. The test plasmids were transformed into the yeast strain as described (Ito et al. 1983) and transformants plated on minimal medium containing glucose but lacking three nutrients, histidine, tryptophan, and uracil, which selected for pGilda, $\mathrm{pB} 42 \mathrm{AD}$, and p8op-lacZ plasmids, respectively. To test for potential interactions the transformants are cultured in induction medium containing galactose and raffinose and assayed for expression of $\beta$-galactosidase as described below. In addition, to test for expression of the LEU2 gene, transformants were plated on the same medium with or without leucine.

TABLE 1. List of mutagenic primers used to construct rpS4 and rpS5 mutants

\begin{tabular}{|c|c|c|c|c|}
\hline Mutant & Mutation & Primers $^{\mathrm{a}}$ & Phenotype & Reference \\
\hline \multicolumn{5}{|c|}{$r p s D$ (rpS4) mutants } \\
\hline Q53L & $\mathrm{CAG} \rightarrow \mathrm{CTG}$ & $\begin{array}{l}\text { ctgtctgactatggtgtgCTGttgcgtgaa } \\
\text { ttcacgcaaCAGcacaccatagtcagacag }\end{array}$ & $\begin{array}{l}\text { res in S. typhimurium; } \\
\text { ram in E. coli }\end{array}$ & Bjorkman et al. (1999) \\
\hline Q53P & $\mathrm{CAG} \rightarrow \mathrm{CCG}$ & $\begin{array}{l}\text { ctatggtgtgCCGttgcgtgaaaagc } \\
\text { gcttttcacgcaaCGGcacaccatag }\end{array}$ & ram in E. coli & Bjorkman et al. (1999) \\
\hline H197A & $\mathrm{CAC} \rightarrow \mathrm{GCC}$ & $\begin{array}{l}\text { gacattaacgaaGCCctgatcgtcgag } \\
\text { ctcgacgatcagGGCttcgttaatgtc }\end{array}$ & ram? & Ogle et al. 2002 \\
\hline $1199 \mathrm{~N}$ & $\mathrm{ATC} \rightarrow \mathrm{AAC}$ & $\begin{array}{l}\text { cgaacacctgAACgtcgagctttac } \\
\text { gtaaagctcgacGTTcaggtgttcg }\end{array}$ & ram in S. typhimurium & Bjorkman et al. (1999) \\
\hline$\Delta \mathrm{V} 200$ & $\Delta \mathrm{GTC}$ & $\begin{array}{l}\text { Cgaacacctgatcgagctttactcc } \\
\text { ggagtaaagctcgatcaggtgttcg }\end{array}$ & res in S. typhimurium & Bjorkman et al. (1999) \\
\hline E201* & $\mathrm{GAG} \rightarrow \mathrm{TAG}$ & $\begin{array}{l}\text { cacctgatcgtcTAGctttactccaag } \\
\text { cttggagtaaagCTAgacgatcaggtg }\end{array}$ & res in S. typhimurium & Bjorkman et al. (1999) \\
\hline K205N & $\mathrm{AAG} \rightarrow \mathrm{AAC}$ & $\begin{array}{l}\text { gctttactccAACtaactcgagaagctttgg } \\
\text { ccaaagcttctcgagttaGTTggagtaaagc }\end{array}$ & ram in S. typhimurium & Bjorkman et al. (1999) \\
\hline K205T & $\mathrm{AAG} \rightarrow \mathrm{ACG}$ & $\begin{array}{l}\text { gctttactccACGtaactcgagaagctttgg } \\
\text { ccaaagcttctcgagttaCGTggagtaaagc }\end{array}$ & ram in S. typhimurium & Bjorkman et al. (1999) \\
\hline rpsD-12 & $\Delta 528-532$ & $\begin{array}{l}\text { Ctggaagttgatgctggtggaaggtacgtttaag } \\
\text { cttaaacgtaccttccaccagcatcaacttccag }\end{array}$ & ram in E. coli & $\begin{array}{l}\text { Olsson and Isaksson (1979) } \\
\text { Dahlgren and Ryden-Aulin (2000) }\end{array}$ \\
\hline rpsD-14 & $\begin{array}{l}\text { Insertion of a C } \\
\text { at } 535\end{array}$ & $\begin{array}{l}\text { ctggcaagatggCaaggtacgttta } \\
\text { taaacgtaccttGccatcttgccag }\end{array}$ & ram in E. coli & $\begin{array}{l}\text { Olsson and Isaksson (1979) } \\
\text { Dahlgren and Ryden-Aulin (2000) }\end{array}$ \\
\hline$r p s D-16$ & Trp 169 to UAG & $\begin{array}{l}\text { aaaagccaaccTAGctggaagttga } \\
\text { tcaacttccagCTAggttggctttt }\end{array}$ & ram in E. coli & $\begin{array}{l}\text { Olsson and Isaksson (1979) } \\
\text { Dahlgren and Ryden-Aulin (2000) }\end{array}$ \\
\hline \multicolumn{5}{|c|}{ rpsE (rpS5) mutants } \\
\hline G28D & GGT $\rightarrow$ GAT & $\begin{array}{l}\text { aaaaccgttaaaggtGATcgtattttctccttc } \\
\text { gaaggagaaaatacgATCacctttaacggtttt }\end{array}$ & ram in E. coli & Kirthi et al. (2006) \\
\hline G101D & GGT $\rightarrow$ GAC & $\begin{array}{l}\text { ccggcttccgaaGACaccggtatcatc } \\
\text { gatgataccggtGTCttcggaagccgg }\end{array}$ & $\begin{array}{l}\text { Sm independence in } \\
\text { S. typhimurium }\end{array}$ & Bjorkman et al. (1999) \\
\hline G101E & GGT $\rightarrow$ GAG & $\begin{array}{l}\text { ccggcttccgaaGAGaccggtatcatc } \\
\text { gatgataccggtCTCttcggaagccgg }\end{array}$ & $\begin{array}{l}\text { Unknown; Similar to } \\
\text { G101D? }\end{array}$ & \\
\hline G103R & $\mathrm{GGT} \rightarrow \mathrm{CGT}$ & $\begin{array}{l}\text { ccgaaggtaccCGTatcatcgccgg } \\
\text { ccggcgatgatACGggtaccttcgg }\end{array}$ & ram in E. coli & Olsson and Isaksson (1979) \\
\hline G108S & $\mathrm{GGT} \rightarrow \mathrm{AGC}$ & $\begin{array}{l}\text { catcgccggtAGCgcaatgcgcg } \\
\text { cgcgcattgcGCTaccggcgatg }\end{array}$ & $\begin{array}{l}\text { Fast growing in rpsL } \\
\text { S. typhimurium strain }\end{array}$ & Kirthi et al. (2006) \\
\hline R111C & $\mathrm{CGC} \rightarrow \mathrm{TGC}$ & $\begin{array}{l}\text { ggtggtgcaatgTGCgccgttctgg } \\
\text { ccagaacggcGCAcattgcaccacc }\end{array}$ & $\begin{array}{l}\text { Sm independence } \\
\text { S. typhimurium }\end{array}$ & Kirthi et al. (2006) \\
\hline $\mathrm{R} 111 \mathrm{~L}$ & $\mathrm{CGC} \rightarrow \mathrm{CTC}$ & $\begin{array}{l}\text { ggtggtgcaatgCTCgccgttctgg } \\
\text { ccagaacggcGAGcattgcaccacc }\end{array}$ & $\begin{array}{l}\text { ram in E. coli; ram in } \\
\text { S. typhimurium }\end{array}$ & $\begin{array}{l}\text { Kirthi et al. (2006) } \\
\text { Itoh and Wittman (1973) }\end{array}$ \\
\hline
\end{tabular}

${ }^{\mathrm{a}}$ Mutations are shown in capital letters. 


\section{Plasmid construction and mutagenesis}

The gene encoding rpS4 ( $r p s D)$ was amplified using polymerase chain reaction using the primers, $5^{\prime}$-GGAGGCGAATTCATGGCA AGATATTTGGGTCCTAAGCTCAAG-3', and 5' - GGCCAGCTC GAGTTACTTGGAGTAAAGCTCGACGATCAGGTGTTCG-3' (restriction sites underlined) and ligated into pB42AD at EcoR1 and XhoI restriction sites. Similarly, $r p s E$ encoding rpS5 is amplified using primers, 5'-GGCCGCGGATCCATGGCTCACATCGAAAA ACAAGCTGGCGA-3' ${ }^{\prime}$ and $5^{\prime}$-GCGGCACTCGAGTTATTTCCCA GAATTTCTTCAACGGATT- ${ }^{\prime}$ and cloned into BamHI and XhoI sites of pGilda. The rpsE gene was fused to LexA of pGilda by deleting the A of the start codon, ATG, by site-directed mutagenesis using primers $5^{\prime}$-GGGGATCCTGGCTCACATCG-3' and $5^{\prime}$-CGATGTGAGCCAGGATCCCCGGG-3'. Mutations in $r p s D$ and $r p s E$ are constructed using mutagenic oligonucleotides (Table 1) using QuikChange mutagenesis (Stratagene). After confirming that the clones had the mutations by DNA sequencing (ABI), all of the mutant constructs were subcloned back into the parent plasmid to eliminate any secondary mutations created by the PCR mutagenesis. The presence of mutations is confirmed by sequencing $(\mathrm{ABI})$ using pGilda sequencing primer $\left(5^{\prime}\right.$-CGTCAGC AGAGCTTCACCATT- $\left.3^{\prime}\right)$ and $\mathrm{pB} 42 \mathrm{AD}$ sequencing primer $\left(5^{\prime}\right.$ CCTGACTGGCTGAAATCGAATGGT-3').

\section{$\beta$-Galactosidase assays}

Three to four clones of each transformant were grown in glucose minimal media lacking histidine, tryptophan, and uracil to saturation. The saturated cultures were washed three times in similar medium with galactose/raffinose as carbon source and were finally resuspended in the same amount of galactose/raffinose medium. One tenth of the washed cells was incubated for $3-5 \mathrm{~h}$, to an $\mathrm{OD}_{600}$ of $\sim 0.8-1.0$. The activity of $\beta$-galactosidase were determined essentially as described (Farabaugh et al. 1993). The significance of differences in activities among the mutants tested was determined by ANOVA followed by Tukey's post hoc test; all statistical tests were performed using KaleidoGraph (Synergy Software).

\section{Protein identification by Western blotting}

Western blotting was used to confirm expression of the fusion proteins. Transformed cells were grown to saturation, washed, and diluted 50- to 100-fold into galactose/raffinose minimal medium, and allowed to grow to an $\mathrm{OD}_{600}$ of 0.8 to 1 . The cells were washed and resuspended in lysis buffer $(25 \mathrm{mM}$ Tris- $\mathrm{HCl}$ at $\mathrm{pH} 7.5,1 \mathrm{mM}$ EDTA, $0.1 \%$ Triton X-100, $0.15 \mathrm{M} \mathrm{NaCl}, 1 \mathrm{mM}$ DTT, and Halt Protease Inhibitor Cocktail $[10 \mu \mathrm{L} / \mathrm{mL}])$ and lysed by vortexing with glass beads. The protein extracts were quantified by Bradford assay (Sigma). Equivalent amounts of extract proteins were separated by SDS-PAGE. All the constructs in pGilda were detected using anti-LexA antibody (Invitrogen; 1:10000) and those in $\mathrm{pB} 42 \mathrm{AD}$ with anti-HA antibody (Invitrogen; $1-2 \mu \mathrm{g} / \mathrm{mL}$ ). $\beta$-Tubulin used as a loading control was visualized by a specific antibody (ANASPEC; 1:200). Visualization of Western blots was performed using the ECL Western Blotting Substrate Kit (Pierce).

\section{Coimmunoprecipitation assays}

Overnight cultures of yeast transformants grown in glucose minimal medium were washed thrice times with same volume of galactose/raffinose minimal medium, diluted 10 - to 50 -fold into the same medium and incubated for 4 to $5 \mathrm{~h}\left(\mathrm{OD}_{600} 0.8-1.0\right)$. The cells were harvested, resuspended in ice-cold lysis buffer $(25$ $\mathrm{mM}$ Tris- $\mathrm{HCl}$ at $\mathrm{pH} 7.5,1 \mathrm{mM}$ EDTA, $0.1 \%$ Triton X-100, $0.15 \mathrm{M}$ $\mathrm{NaCl}, 1 \mathrm{mM}$ DTT, and Halt Protease Inhibitor Cocktail [13 $\mu \mathrm{L} /$ $\mathrm{mL}]$ ) and lysed by vortexing with glass beads. The supernatant was collected after spinning the lysates for $5 \mathrm{~min}$ at $4^{\circ} \mathrm{C}$. The total amount of protein in the extracts is estimated by Bradford assay (Sigma). Lysates with equivalent amounts of protein are subjected to coimmunoprecipitation assays using Profound HA Tag IP/COIP Kit (Pierce) according to the manufacturer's instructions. Briefly, anti-HA agarose slurry $(6 \mu \mathrm{L})$ was incubated with cell lysate in a spin column overnight at $4^{\circ} \mathrm{C}$. The columns were washed four times with TBS-T $(25 \mathrm{mM}$ Tris- $\mathrm{HCl}, 0.15 \mathrm{M} \mathrm{NaCl}$ [pH 7.2], and $0.05 \%$ Tween-20) by pulse centrifuging for $10 \mathrm{sec}$ at $5000 \mathrm{rpm}$ each time and the HA-tagged proteins were eluted using $25 \mu \mathrm{L}$ of nonreducing sample buffer. The eluted proteins were prepared for reducing SDS-PAGE by adding $\beta$-mercaptoethanol and detected by Western blotting as described previously. The final washes are also subjected to SDS-PAGE by adding $2 \times$ sample buffer (100 mM Tris- $\mathrm{HCl}$ at pH 6.8, 4\% SDS, 20\% glycerol, $0.2 \%$ bromophenol blue, and 5\% $\beta$-mercaptoethanol). Wherever necessary, the blots were reprobed with a second antibody after incubating with Stripping Buffer (Restore Plus Western blot Stripping buffer from Pierce) for $15 \mathrm{~min}$ at room temperature. The quantity of protein precipitated was determined using the Image J software program (Research Services Branch, National Institute of Mental Health).

\section{ACKNOWLEDGMENT}

This work was supported by grants from the National Institute of General Medical Science to P.J.F. (GM029480).

Received December 22, 2008; accepted February 24, 2009.

\section{REFERENCES}

Allen, P.N. and Noller, H.F. 1989. Mutations in ribosomal proteins S4 and S12 influence the higher order structure of $16 \mathrm{~S}$ ribosomal RNA. J. Mol. Biol. 208: 457-468.

Allen, P.N. and Noller, H.F. 1991. A single base substitution in $16 \mathrm{~S}$ ribosomal RNA suppresses streptomycin dependence and increases the frequency of translational errors. Cell 66: 141-148.

Birge, E.A. and Kurland, C.G. 1969. Altered ribosomal protein in streptomycin-dependent Escherichia coli. Science 166: 12821284.

Birge, E.A. and Kurland, C.G. 1970. Reversion of a streptomycindependent strain of Escherichia coli. Mol. Gen. Genet. 109: 356369.

Bjorkman, J., Samuelsson, P., Andersson, D.I., and Hughes, D. 1999. Novel ribosomal mutations affecting translational accuracy, antibiotic resistance and virulence of Salmonella typhimurium. Mol. Microbiol. 31: 53-58.

Brodersen, D.E., Clemons Jr., W.M., Carter, A.P., Wimberly, B.T., and Ramakrishnan, V. 2002. Crystal structure of the 30 S ribosomal subunit from Thermus thermophilus: Structure of the proteins and their interactions with 16 S RNA. J. Mol. Biol. 316: 725-768.

Carter, A.P., Clemons, W.M., Brodersen, D.E., Morgan-Warren, R.J., Wimberly, B.T., and Ramakrishnan, V. 2000. Functional insights from the structure of the $30 \mathrm{~S}$ ribosomal subunit and its interactions with antibiotics. Nature 407: 340-348. 
Dahlgren, A. and Ryden-Aulin, M. 2000. A novel mutation in ribosomal protein S4 thataffects the function of a mutated RF1. Biochimie 82: 683-691.

Davies, J., Gilbert, W., and Gorini, L. 1964. Streptomycin, suppression, and the code. Proc. Natl. Acad. Sci. 51: 883-890.

Daya-Grosjean, L., Garrett, R.A., Pongs, O., Stoffler, G., and Wittmann, H.G. 1972. Properties of the interaction of ribosomal protein S4 and 16S RNA in Escherichia coli revertants from Streptomycin dependence to independence. Mol. Gen. Genet. 119: $277-286$

Deusser, E., Stoffler, G., and Wittmann, H.G. 1970. Ribosomal proteins. XVI. Altered S4 proteins in Escherichia coli revertants from streptomycin dependence to independence. Mol. Gen. Genet. 109: 298-302.

Donner, D. and Kurland, C.G. 1972. Changes in the primary structure of a mutationally altered ribosomal protein S4 of Escherichia coli. Mol. Gen. Genet. 115: 49-53.

Farabaugh, P., Vimaladithan, A., Türkel, S., Johnson, R., and Zhao, H. 1993. Three downstream sites repress transcription of a Ty2 retrotransposon in Saccharomyces cerevisiae. Mol. Cell. Biol. 13: 2081-2090.

Gorini, L. and Kataja, E. 1964. Phenotypic repair by streptomycin of defective genotypes in E. coli. Proc. Natl. Acad. Sci. 51: 487-493.

Gromadski, K.B., Daviter, T., and Rodnina, M.V. 2006. A uniform response to mismatches in codon-anticodon complexes ensures ribosomal fidelity. Mol. Cell 21: 369-377.

Grosjean, H.J., de Henau, S., and Crothers, D.M. 1978. On the physical basis for ambiguity in genetic coding interactions. Proc. Natl. Acad. Sci. 75: 610-614.

Hopfield, J.J. 1974. Kinetic proofreading: A new mechanism for reducing errors in biosynthetic processes requiring high specificity. Proc. Natl. Acad. Sci. 71: 4135-4139.

Ibba, M. and Soll, D. 1999. Quality control mechanisms during translation. Science 286: 1893-1897.

Inaoka, T., Kasai, K., and Ochi, K. 2001. Construction of an in vivo nonsense readthrough assay system and functional analysis of ribosomal proteins S12, S4, and S5 in Bacillus subtilis. J. Bacteriol. 183: 4958-4963.

Ito, H., Fukuda, Y., Murata, K., and Kimura, A. 1983. Transformation of intact yeast cells treated with alkali cations. J. Bacteriol. 153: 163-168.

Ito, T. and Wittmann, H.G. 1973. Amino acid replacements in proteins S5 and S12 of two Escherichia coli revertant from streptomycin dependence to independence. Mol. Gen. Genet. 127: 19-32.

Kirthi, N., Roy-Chaudhuri, B., Kelley, T., and Culver, G.M. 2006. A novel single amino acid change in small subunit ribosomal protein S5 has profound effects on translational fidelity. RNA 12: 2080 2091.

Kramer, E.B. and Farabaugh, P.J. 2007. The frequency of translational misreading errors in E. coli is largely determined by tRNA competition. RNA 13: 87-96.

Kreider, G. and Brownstein, B.L. 1971. A mutation suppressing streptomycin dependence. II. An altered protein on the $30 \mathrm{~S}$ ribosomal subunit. J. Mol. Biol. 61: 135-142.

Kurland, C., Hughes, D., and Ehrenberg, M. 1996. Limitations of translational accuracy. In Escherichia coli and Salmonella: Cellular and molecular biology (eds. F.C. Neidhardt et al.), pp. 979-1004. ASM Press, Washington, DC.

Leontis, N.B., Stombaugh, J., and Westhof, E. 2002. The non-WatsonCrick base pairs and their associated isostericity matrices. Nucleic Acids Res. 30: 3497-3531.
Ma, J. and Ptashne, M. 1987. A new class of yeast transcriptional activators. Cell 51: 113-119.

Maisnier-Patin, S., Berg, O.G., Liljas, L., and Andersson, D.I. 2002. Compensatory adaptation to the deleterious effect of antibiotic resistance in Salmonella typhimurium. Mol. Microbiol. 46: 355-366.

Moazed, D. and Noller, H.F. 1990. Binding of tRNA to the ribosomal $\mathrm{A}$ and $\mathrm{P}$ sites protects two distinct sets of nucleotides in 16S rRNA. J. Mol. Biol. 211: 135-145.

Ninio, J. 1975. Kinetic amplification of enzyme discrimination. Biochimie 57: 587-595.

O'Connor, M., Brunelli, C.A., Firpo, M.A., Gregory, S.T., Lieberman, K.R., Lodmell, J.S., Moine, H., Van Ryk, D.I., and Dahlberg, A.E. 1995. Genetic probes of ribosomal RNA function. Biochem. Cell Biol. 73: 859-868.

O'Connor, M., Thomas, C., Zimmermann, R., and Dahlberg, A. 1997. Decoding fidelity at the ribosomal $\mathrm{A}$ and $\mathrm{P}$ sites: Influence of mutations in three different regions of the decoding domain in $16 \mathrm{~S}$ rRNA. Nucleic Acids Res. 25: 1185-1193.

Ogle, J.M. and Ramakrishnan, V. 2005. Structural insights into translational fidelity. Annu. Rev. Biochem. 74: 129-177.

Ogle, J.M., Brodersen, D.E., Clemons Jr., W.M., Tarry, M.J., Carter, A.P., and Ramakrishnan, V. 2001. Recognition of cognate transfer RNA by the 30 S ribosomal subunit. Science 292: $897-$ 902.

Ogle, J.M., Murphy, F.V., Tarry, M.J., and Ramakrishnan, V. 2002. Selection of tRNA by the ribosome requires a transition from an open to a closed form. Cell 111: 721-732.

Olsson, M.O. and Isaksson, L.A. 1979. Analysis of rpsD mutations in Escherichia coli. I. Comparison of mutants with various alterations in ribosomal protein S4. Mol. Gen. Genet. 169: 251-257.

Ozaki, M., Mizushima, S., and Nomura, M. 1969. Identification and functional characterization of the protein controlled by the streptomycin-resistant locus in E. coli. Nature 222: 333-339.

Potapov, A.P. 1982. A stereospecific mechanism for the aminoacyltRNA selection at the ribosome. FEBS Lett. 146: 5-8.

Rodnina, M.V., Gromadski, K.B., Kothe, U., and Wieden, H.-J. 2005. Recognition and selection of tRNA in translation. FEBS Lett. 579: 938-942.

Rosset, R. and Gorini, L. 1969. A ribosomal ambiguity mutation. J. Mol. Biol. 39: 95-112.

Ruusala, T., Andersson, D., Ehrenberg, M., and Kurland, C.G. 1984. Hyper-accurate ribosomes inhibit growth. EMBO J. 3: 2575-2580.

Stoffler, G., Deusser, E., Wittmann, H.G., and Apirion, D. 1971. Ribosomal proteins. XIX. Altered S5 ribosomal protein in an Escherichia coli revertant from strptomycin dependence to independence. Mol. Gen. Genet. 111: 334-341.

Thompson, R.C. 1988. EFTu provides an internal kinetic standard for translational accuracy. Trends Biochem. Sci. 13: 91-93.

Thompson, R.C. and Stone, P.J. 1977. Proofreading of the codonanticodon interaction on ribosomes. Proc. Natl. Acad. Sci. 74: 198202.

Uhlenbeck, O.C., Martin, F.H., and Doty, P. 1971. Self-complementary oligoribonucleotides: Effects of helix defects and guanylic acidcytidylic acid base pairs. J. Mol. Biol. 57: 217-229.

Wimberly, B.T., Brodersen, D.E., Clemons Jr., W.M., MorganWarren, R.J., Carter, A.P., Vonrhein, C., Hartsch, T., and Ramakrishnan, V. 2000. Structure of the 30 S ribosomal subunit. Nature 407: 327-339.

Zengel, J.M., Young, R., Dennis, P.P., and Nomura, M. 1977. Role of ribosomal protein S12 in peptide chain elongation: Analysis of pleiotropic, streptomycin-resistant mutants of Escherichia coli. J. Bacteriol. 129: 1320-1329. 

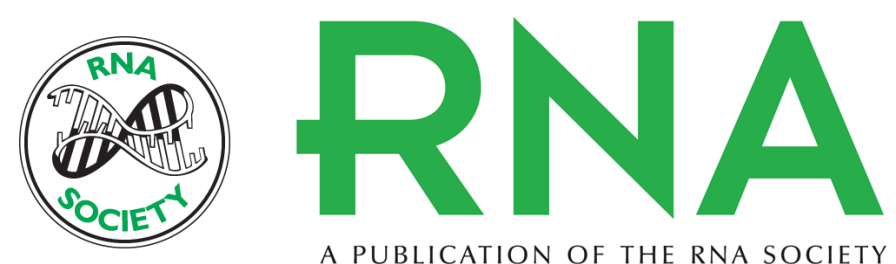

A PUBLICATION OF THE RNA SOCIETY

\section{Accuracy modulating mutations of the ribosomal protein S4-S5 interface do not necessarily destabilize the rps4-rps5 protein-protein interaction}

Haritha Vallabhaneni and Philip J. Farabaugh

RNA 2009 15: 1100-1109 originally published online April 22, 2009

Access the most recent version at doi:10.1261/rna.1530509

References This article cites 46 articles, 14 of which can be accessed free at:

http://rnajournal.cshlp.org/content/15/6/1100.full.html\#ref-list-1

\section{License}

Email Alerting Receive free email alerts when new articles cite this article - sign up in the box at the Service top right corner of the article or click here.

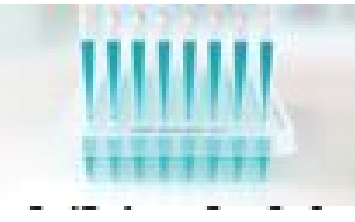

Providing Precise Solutions for your research.

To subscribe to RNA go to:

http://rnajournal.cshlp.org/subscriptions 